\title{
Produtividade e principais problemas fitossanitários de cultivares de batata em Sergipe
}

\author{
Maria Urbana Corrêa Nunes \\ Embrapa Tabuleiros Costeiros, C. Postal 44, 49.001-970 Aracaju-SE. E-mail: murbana@cpatc.embrapa.br
}

\section{RESUMO}

Sete cultivares de batata (Solanum tuberosum L.) foram avaliadas durante três anos em Itabaiana-SE, com o objetivo de verificar a potencialidade de produção à altitude de $180 \mathrm{~m}$ e identificar os problemas fitossanitários que afetam a produtividade. Os experimentos foram conduzidos nos meses de junho a setembro, utilizando-se o delineamento experimental de blocos ao acaso, com cinco repetições. Após a colheita foram avaliadas a produção total, comercial (tubérculos sadios e com peso mínimo de $40 \mathrm{~g}$ ) e perdas de produção decorrentes de sarna, podridão-mole, nematóide e insetos do solo. A análise conjunta das produções totais e comerciais de tubérculos revelou que as cultivares mais produtivas foram Baronesa $(33,7 \mathrm{t}$ / ha), Monalisa (32,7 t/ha) e Elvira (31,4 t/ha). A produção comercial variou de 3,4 t/ha a 22,5 t/ha, destacando-se as cvs. Elvira e Monalisa, com rendimentos de 22,5 t/ha e 20,5 t/ha respectivamente, com apenas três pulverizações com defensivos. Os problemas fitossanitários que mais afetaram a cv. Elvira foram sarna $(13,1 \%)$ e nematóide das galhas ( $7,2 \%)$, enquanto a 'Monalisa' teve sua produção prejudicada mais pela incidência de insetos $(15,1 \%)$, sarna $(12,0 \%)$ e nematóide $(9,7 \%)$. A cv. Monalisa apresentou tubérculos com melhores características para comercialização e está sendo cultivada a nível comercial em substituição às variedades Bintje e Baraka anteriormente cultivadas na região. Os resultados indicam a potencialidade da região de Itabaiana para a produção de batata.

Palavras-chave: Solanum tuberosum, adaptação, doenças, inseto, nematóide.

\begin{abstract}
Yield and phytosanitary problems of potato cultivars in Sergipe, Brazil

The yield potential and main diseases affecting productivity of seven potato (Solanum tuberosum L.) cvs. were evaluated during a three years period in experiments carried out at low altitude conditions of Sergipe State, Brazil. The experimental plots were located at $180 \mathrm{~m}$ of sea level in a Red-Yellow Podzolic sandy-clay texture soil. The experimental design was of complete randomized blocks with five replications. Tubers were sown yearly in June and harvested in September. After harvest, total yield, commercial yield (healthy tubers with $40 \mathrm{~g}$ minimum weight) and losses caused by scab, soft-rot, nematodes, and soil insects were evaluated. The analysis of variance for total and commercial yield of tubers highlighted Baronesa (33.7 $\mathrm{kg} / \mathrm{ha})$, Monalisa $(32.7 \mathrm{~kg} / \mathrm{ha})$, and Elvira $(31.4 \mathrm{~kg} / \mathrm{ha})$ as the most productive cvs. The commercial yields varied from $3.4 \mathrm{t} / \mathrm{ha}$ to $22 \mathrm{at} /$ ha. The cvs. Elvira and Monalisa, yielded $22.5 \mathrm{~kg} / \mathrm{ha}$ and $20.5 \mathrm{~kg} / \mathrm{ha}$, respectively. Only three insecticides applications were necessary. Scab $(13.1 \%)$ and nematode $(7.2 \%)$ were the main phytosanitary problems affecting Elvira cv. In relation to Monalisa, pin larvae soil insect $(15.1 \%)$, scab $(12.0 \%)$, and nematode $(9.7 \%)$ were the main causes of yield loss. Because of its best commercial tuber characteristics Monalisa has been planted instead of Bintje and Baraka, both commonly used in the region. The results indicate the suitability of Itabaiana region for potato production.
\end{abstract}

Keywords: Solanum tuberosum, adaptation, disease, insect, nematode.

(Recebido para publicação em 09 de dezembro de 1999 e aceito em 06 de junho de 2002)

\begin{abstract}
A batata (Solanum tuberosum L.) é uma das hortaliças de maior aceitação pelo consumidor, mesmo em regiões onde as condições de clima e solo não sejam ideais para o desenvolvimento vegetativo e reprodutivo desta cultura. Maiores produtividades são alcançadas nos estados de Goiás, Distrito Federal e Minas Gerais com médias de 46,0, 30,7 e 20,8 t/ha, respectivamente. O estado de Sergipe apresenta produtividade média de $9,2 \mathrm{t} / \mathrm{ha}$, superior aos estados do Ceará, Paraíba, Pernambuco e Santa Catarina e inferior à média brasileira (14,6 t/ha) (Anuário Estatístico do Brasil, 1995).

No Brasil, o cultivo de batata sempre foi recomendado em microclimas de altitude, mas tal paradigma foi mudado,
\end{abstract}

pelo rendimento da cultura em regiões de baixa altitude, como Sergipe, onde destacam-se os municípios de Itabaiana e Simão Dias, com altitudes de $180 \mathrm{~m}$ e $250 \mathrm{~m}$, respectivamente. A área plantada, todavia, está estagnada desde a década de 80 entre 45 e 55 ha, com um pico de 125 ha em 1990. O estado ainda é um dos principais importadores de batata destinada ao abastecimento (Anuário Estatístico de Sergipe, 1996). Embora exista mercado consumidor, áreas potenciais para o cultivo, interesse dos agricultores por se tratar de uma cultura que apresenta retorno econômico, a bataticultura vem se desenvolvendo em pequena escala, com fases de oscilação, tanto em área plantada quanto em rendimento, com o cultivo de Bintje e Baraka.
Um dos fatores primordiais para a expansão dessa cultura no Sergipe é a identificação de cultivares mais adaptadas às condições climáticas locais, que combinem maior produtividade com melhor qualidade, uma vez que o clima desempenha papel importante no comportamento de diversas cultivares (Antunes \& Fortes, 1981). Além disso, é necessário conhecer os principais problemas fitossanitários da cultura no ambiente de cultivo. Nesse aspecto, devem-se considerar a adaptação da cultivar ao tipo de solo da região, a suscetibilidade às pragas e doenças causadas por fungos, bactérias, vírus e nematóides e aos distúrbios fisiológicos, como embonecamento e rachaduras, além do formato e coloração do tubérculo (Boock, 1975). 
A influência desses diversos fatores no comportamento de cultivares, com reflexos na produção, é evidenciada por trabalhos de pesquisa desenvolvidos em diferentes regiões do Brasil. Pelos resultados obtidos nos ensaios nacionais de batata no período de 1980/82, as cvs. Elvira e Santo Amor se destacaram em produtividade em diferentes ambientes (Embrapa, 1983). As cvs. Elvira e Santo Amor foram as mais adaptadas, tanto nas condições climáticas de Conceição do Castelo (ES) (Neto et al.,1982) como em Jaboticabal, apresentando as maiores produções de tubérculos comercializáveis (Churata-Masca \& Storion, 1984). Silva (1992) constatou que 'Santo Amor', 'Baronesa' e 'Baraka' foram as mais produtivas no litoral catarinense. Em estudo do comportamento de 26 cvs., em Anápolis, sobressaíram-se Elvira (30,5 t/ha), Aracy (28,8 t/ha) e Baronesa $(28,4 \mathrm{t} / \mathrm{ha})$ (Filgueira \& Câmara, 1984). Entre as cvs. recomendadas para Mato Grosso do Sul, estão Aracy, Baronesa e Monalisa, como mais produtivas e com disponibilidade de batata-semente no mercado nacional (Zatarim \& Leonel, 1990). No estado do Piauí, a $250 \mathrm{~m}$ de altitude, Neto et al. (1991) obtiveram maiores produtividades com 'Baraka' (24,1 t/ha) e 'Baronesa' (23,3 t/ha). Na Paraíba, 'Baraka' e 'Monalisa' são as mais adaptadas, por apresentarem maior rusticidade e rendimento (Lopes et al., 1996).

Com a visão de reduzir a importação de batata pelo estado de Sergipe e criar alternativa de diversificação de culturas para os agricultores, os objetivos do presente trabalho foram verificar a potencialidade da produção de batata nas condições de baixa altitude; identificar problemas fitossanitários que afetam a cultura e selecionar cultivares mais adaptadas às condições locais.

\section{MATERIAL E MÉTODOS}

Os experimentos foram conduzidos na área experimental localizada no povoado Água Branca, município de Itabaiana (SE), à altitude de $180 \mathrm{~m}$, em solo Podzólico Vermelho-Amarelo, profundo, de textura areno-argilosa e de média fertilidade.

Foram avaliadas em 1995, 1996 e 1997 as cvs. Baronesa, Baraka, Elvira,

Tabela 1. Produção total e comercial de sete cultivares de batata na região de baixa altitude do estado de Sergipe. Itabaiana - SE, 1995-1997(1).

\begin{tabular}{lcc}
\hline \multicolumn{1}{c}{ Cultivar } & $\begin{array}{c}\text { Produção total } \\
\text { (t/ha) }\end{array}$ & $\begin{array}{c}\text { Produção comercial } \\
\text { (t/ha) }\end{array}$ \\
\hline Baronesa & $33,67 \mathrm{a}$ & $14,52 \mathrm{C}$ \\
Monalisa & $32,73 \mathrm{a}$ & $20,54 \mathrm{~b}$ \\
Elvira & $31,43 \mathrm{a}$ & $22,47 \mathrm{a}$ \\
Baraka & $31,33 \mathrm{ab}$ & $3,35 \mathrm{f}$ \\
Trapeira & $31,19 \mathrm{ab}$ & $9,06 \mathrm{e}$ \\
Santo Amor & $30,96 \mathrm{ab}$ & $15,52 \mathrm{c}$ \\
Monte Bonito & $28,25 \mathrm{~b}$ & $12,48 \mathrm{~d}$ \\
\hline C. V. (\%) & 8,86 & 8,45 \\
\hline
\end{tabular}

(1) Médias seguidas pela mesma letra, na coluna, não diferem entre si a 5\% de probabilidade pelo teste de Tukey.

Monte Bonito, Monalisa, Santo Amor e Trapeira, com plantios realizados na primeira quinzena de junho e colheita em setembro. As parcelas experimentais, arranjadas no delineamento de blocos casualizados, com cinco repetições, constaram de 40 plantas dispostas em quatro fileiras no espaçamento de $0,80 \mathrm{~m}$ entre fileiras e $0,40 \mathrm{~m}$ entre plantas, considerando como úteis as 16 plantas centrais. Com base nos resultados da análise de solo, aplicou-se no plantio o esterco bovino distribuído a lanço (30 t/ha) e adubo químico, 1,250 t/ha da fórmula 6-24-12 enriquecida com $\mathrm{Ca}, \mathrm{S}, \mathrm{Zn}, \mathrm{B}, \mathrm{Cu}, \mathrm{Fe}$, Mn e Mo, aplicados no sulco de plantio. A adubação de cobertura foi realizada, por ocasião da amontoa, aos 30 dias após o plantio, usando $60 \mathrm{~kg} / \mathrm{ha} \mathrm{de}$ $\mathrm{N}$ na forma de sulfato de amônio. Foram realizadas duas capinas e uma amontoa, e não foi feita irrigação complementar à chuva. A precipitação pluvial total nesse período foi de $775 \mathrm{~mm}$, $509 \mathrm{~mm}$ e $729 \mathrm{~mm}$, em 1995, 1996 e 1997, respectivamente. A temperatura média de maio a agosto dos três anos oscilou de $22^{\circ} \mathrm{C}$ a $25,1^{\circ} \mathrm{C}$. Foram feitas apenas duas pulverizações com fungicida à base de oxicloreto de cobre e uma com o inseticida cyromazine. A colheita foi realizada após a senescência de pelo menos $80 \%$ das plantas, o que ocorreu entre 80 e 90 dias após o plantio. Todas as cultivares foram avaliadas quanto à produção total e comercial (tubérculos sadios e com peso acima de 40 g) e perda de produção causada por sarna (Streptomyces spp.), podridão-mole
(Erwinia spp.), nematóide (Meloidogyne spp.) ou insetos [larva-alfinete (Diabrotica spp.) e emboá], considerando aqueles que apresentassem qualquer lesão da doença ou qualquer perfuração por inseto. Essas perdas foram determinadas pela pesagem dos tubérculos danificados e calculada a percentagem em relação à produção total.

\section{RESULTADOS E DISCUSSÃO}

A análise conjunta dos rendimentos totais e comerciais de tubérculos obtidos nos três anos de plantio mostrou diferenças significativas entre as cultivares (Tabela 1). Quanto à produção total, as cvs. Baronesa, Monalisa, Elvira, Baraka, Trapeira e Santo Amor não diferiram entre si. Contudo, as três primeiras cvs. superaram significativamente a 'Monte Bonito' que apresentou menor média de produção. $\mathrm{O}$ destaque da $\mathrm{Ba}$ ronesa entre as melhores cultivares foi observado também em Santa Catarina (Silva, 1992), em Anápolis, à altitude de 970 m (Filgueira \& Câmara, 1984), no Piauí, à altitude de $250 \mathrm{~m}$ (Neto et al., 1991), e no Mato Grosso do Sul, à altitude média de $530 \mathrm{~m}$ (Zatarin \& Leonel, 1990), o que sugere alto potencial de adaptação dessa cultivar aos diversos ambientes. Silva, (1992), Embrapa (1983) e Neto et al. (1982) encontraram valores semelhantes aos obtidos neste trabalho quanto à produção total da cultivar Santo Amor.

Em produção comercial, a 'Elvira' superou as demais, com produção superior à encontrada por Neto et al. 
Tabela 2. Perdas de produção causadas por sarna, podridão-mole, nematóide das galhas e insetos de sete cultivares de batata em condições de baixa altitude no Estado de Sergipe, Itabaiana - SE, 1995 a $1997^{(1)}$.

\begin{tabular}{lccccccc}
\hline Cultivares & Sarna (\%) & & Podridão-mole (\%) & Nematóide (\%) & Inseto (\%) & \\
\hline Baronesa & 3,19 & $\mathrm{e}$ & $5,72 \mathrm{a}$ & $35,56 \mathrm{a}$ & 6,08 & $\mathrm{~b}$ \\
Monalisa & 11,98 & $\mathrm{~d}$ & $4,53 \mathrm{a}$ & 9,74 & $\mathrm{~d}$ & $15,05 \mathrm{a}$ & \\
Elvira & 13,05 & $\mathrm{~d}$ & $3,51 \mathrm{a}$ & 7,15 & $\mathrm{~d}$ & 3,16 & $\mathrm{c}$ \\
Baraka & $83,86 \mathrm{a}$ & $3,07 \mathrm{a}$ & 0,88 & $\mathrm{e}$ & 0,71 & $\mathrm{~d}$ \\
Trapeira & $41,08 \mathrm{~b}$ & $5,88 \mathrm{a}$ & 14,28 & $\mathrm{C}$ & 5,76 & $\mathrm{~b}$ \\
Santo Amor & $23,65 \mathrm{C}$ & $2,39 \mathrm{a}$ & 16,29 & $\mathrm{c}$ & 3,83 & $\mathrm{c}$ \\
Monte Bonito & 14,80 & $\mathrm{~d}$ & $7,90 \mathrm{a}$ & 23,60 & $\mathrm{~b}$ & 3,64 & $\mathrm{c}$ \\
\hline C. V. (\%) & 9,20 & 33,87 & 22,74 & & 28,35 & \\
\hline
\end{tabular}

${ }^{(1)}$ Médias seguidas pela mesma letra, na coluna, não diferem entre si a 5\% de probabilidade pelo teste de Tukey.

(1982) (19,8 t/ha) em Domingos Martins (ES). Comportamento semelhante ao da 'Elvira' em Sergipe foi observado também por Churata-Masca \& Storion (1984) em Jaboticabal e por Silva et al. (2000) no plantio de inverno no litoral sul-catarinense. A cultivar Monalisa, recomendada por Zatarin \& Leonel (1990), como a mais produtiva nas condições edafoclimáticas do Mato Grosso do Sul, destacou-se neste trabalho como a segunda cultivar em produção de tubérculos comerciais, com resultado semelhante ao constatado por Lopes et al. (1996) na Paraíba. Entre as cultivares avaliadas, Monalisa apresentou tubérculos com melhores características para o mercado local, com formato alongado, película clara e lisa e polpa de coloração creme-clara. Com exceção da Trapeira e Baraka, todas as cultivares superaram a média estadual, e 'Elvira', 'Monalisa', 'Santo Amor' e 'Baronesa' superaram o rendimento médio nacional.

Durante o desenvolvimento da cultura houve ocorrência de pulgão (Myzus persicae), vaquinha (Diabrotica spp.), larva-minadora (Liriomyza spp.), requeima (Phytophthora infestans) e pinta-preta (Alternaria solani). Os problemas fitossanitários ocorridos com a cultura nas condições locais e que causaram danos aos tubérculos, tornando-os sem valor comercial, foram a ocorrência de sarna (Streptomyces spp.), podridão-mole (Erwinia spp.), nematóide das galhas (Meloidogyne spp.) e insetos de solo como larva-alfinete (Diabrótica speciosa) e emboá (Tabela 2). As maiores perdas de produção foram devidas à sarna, exceto em relação à 'Baronesa'. A 'Baraka' foi altamente suscetível a essa doença, com perda de $83,86 \%$ da produção total, resultado que contraria o obtido por Santos et al. (1986), que a consideraram moderadamente suscetível a Streptomyces spp., e concorda com Mariano et al. (1992), que constataram maior infeção por sarna nessa cultivar, nos estados de Pernambuco e Paraíba. Esse resultado evidencia a baixa adaptação dessa cultivar e a necessidade de sua substituição por outras mais adaptadas, em áreas onde a utilizam.

A 'Elvira', que apresentou a maior produção comercial, teve como principais problemas a sarna e o nematóide das galhas, que podem ser disseminados tanto pela batata-semente como pelo solo (Dias \& Iamauti, 1997). Em relação ao nematóide das galhas, essa cultivar foi menos suscetível que Trapeira, Santo Amor, Baronesa e Monte Bonito, sendo as duas últimas as mais suscetíveis.

Os resultados deste trabalho indicam Elvira como a cultivar mais adaptada à microrregião de Itabaiana, em razão de sua rusticidade. Fato importante a se considerar com respeito à 'Monalisa', é que apesar de se destacar em segundo lugar quanto à produtividade comercial, apresenta tubérculos de formato alongado, com maior aceitação em relação ao tubérculo arredondado da 'Elvira', além da película clara e lisa, e polpa de coloração creme-clara. Após a conclusão deste trabalho, verificou-se que a 'Monalisa' tem sido plantada pelos agricultores sergipanos, em escala comercial, com produtividade média de $20 \mathrm{t}$ / ha e uso mínimo de agrotóxicos.

Tais resultados evidenciam a potencialidade de Sergipe, mais especificamente da microrregião de Itabaiana, para a produção de batata, e permitem inferir que, com o uso da cultivar mais adaptada e que apresenta tubérculos com características adequadas ao mercado, pode-se alcançar um aumento da produção comercial economicamente significativo.

\section{AGRADECIMENTOS}

Aos técnicos Arnaldo Santos Rodrigues, da Embrapa Tabuleiros Costeiros, e Waltênis Braga Silva, da Empresa de Desenvolvimento Agropecuário de Sergipe, pelas avaliações de campo e de pós-colheita, bem como na difusão dos resultados; ao produtor Antônio de Merêncio, por ceder a área para condução dos experimentos e pelo apoio na difusão dos resultados entre os demais produtores.

\section{LITERATURA CITADA}

ANTUNES, F.Z.; FORTES, M. Exigências climáticas da cultura da batata. Informe Agropecuário, Belo Horizonte, v. 7, n. 76, p. 19-30, 1981.

ANUÁRIO ESTATÍSTICO DO BRASIL. Rio de Janeiro, v. 55, p. 1-32, 1995.

ANUÁRIO ESTATÍSTICO DE SERGIPE. Aracaju, v. 18, p. 1-432, 1996.

BOOCK, J.O. Instruções para a cultura da batatinha. Campinas: IAC, 1975. (IAC - Boletim de Pesquisa, 128).

CHURATA-MASCA, M.G.C.; STORION, J.A. Avaliação de cultivares nacionais e importadas de batata nas condições de Jaboticabal, SP. In: CONGRESSO BRASILEIRO DE OLERICUTULTURA, 24., 1984, Jaboticabal. Resumos... Jaboticabal: SOB, 1984. p. 21.

DIAS, J.A.C.S.; IAMAUTI, M.T. Doenças da batateira. In: KIMATI, H.; AMORIM, L.; FILHO, B.A.; CAMARGO, L.E.A.; REZENDE, J.A.M. Manual de fitopatologia - doenças das plantas cultivadas. 3 ed. São Paulo: Ceres, 1997. p. 137-164. 
EMBRAPA. Centro Nacional de Pesquisa de Hortaliças, Planaltina - DF. Ensaio nacional de cultivares de batata 1980/82, Brasília, 1983. 107 p. (Relatório).

FILGUEIRA, F.A.R.; CÂMARA, F.L.A. Comportamento de cultivares européias e brasileiras de batata, nos periodos seco e chuvoso, em Anápolis - 1980/83, Goiânia: ENGOPA - DDI, 1984. 17 p (EMGOPA - Boletim de Pesquisa, 1).

LOPES, E.B.; SILVA, F.C.P.; MOURA, F.T. Recomendações técnicas para o cultivo da batatinha (Solanum tuberosum L.) no Estado da Paraíba. J. Pessoa: EMEPA-PB, 1996. 61 p. (EMEPA-PB. Circular Técnica, 07)

MARIANO, R.L.R.; BARROS, S.T.; SILVA, I.P.; PADOVAN, I.P. Incidência e severidade da sarna comum da batata nos estados da Paraíba e Pernambuco. Cad. Ômega Univ. Fed. Rural PE, Sér. Agron., Recife, n. 4, p. 59-67, 1992.
NETO, J.P.; CARVALHO,J.H.; SOBRINHO, C.A.; SETUBAL, J.W.; RIBEIRO, V.Q. Introdução e avaliação de cultivares de batata (Solanum tuberosum L.) no município de São João do Piauí In: Relatório Técnico Anual UEPAE/Teresina p. 113-116, 1991.

NETO, A.P.M.A.; SILVA, A.A.; CARMO, C.A.S Avaliação de cultivares de batata (Solanum tuberosum L.) no Estado do Espírito Santo. In: CONGRESSO BRASILEIRO DE OLERICULTURA, 22., 1982, Vitória. ES. Resumos... Vitória: SOB, 1982, p. 52.

SANTOS, M.M.F.B.; ANDRIGUETO, J.R.; CAMARGO, C.P. Descrição de cultivares de batata. Brasília,: Secretaria Nacional de Produção Agropecuária - Coordenadoria de Sementes e Mudas, 1986.40 p.
SILVA, A.C.F; ALTHIFF, D.A.; SOUZA, Z.S.; PEDROZA, J.C.C. Cultivares de batata no Litoral Sul Catarinense - plantios de outono e inverno. Agropecuária Catarinense, v. 13, n. 1, p. 3943, 2000.

SILVA, A.C.F. Avaliação de cultivares de batata no litoral Catarinense. Horticultura Brasileira, Brasília, v. 10, n. 1, p. 34 - 37, 1992.

ZATARIN, M.; LEONEL, L.A.K. A cultura da batata (Solanum tuberosum L.) e seu cultivo em Mato Grosso do Sul. Campo Grande: EMPAER, 1990. 59 p. (EMPAER. Documentos, 35). 